\section{PROSPECTIVE STUDY OF THE ETIOLOGY OF VIRAL ACUTE GASTROENTERITIS WITH RV GENOTYPES IN TWO SEASONS AND NORAVIRUS AS SECOND CAUSE}

\author{
F. Neiva ${ }^{1}$, A. Afonso ${ }^{1}$, F. Branca ${ }^{2}$, H. Antunes ${ }^{1,3}$
}

${ }^{1}$ Gastrenterology, Hepatology and Nutrition Unit, Pediatrics Dpt, Braga Hospital, ${ }^{2}$ Clinical Pathology Dept, Braga Hospital, ${ }^{3}$ Life and Health Sciences

Research Institute (ICVS), School of Health Sciences, University of Minho, Campus de Gualtar, Braga, Portugal

Objective: To determine the viruses and genotypes circulating in children attending to Braga Hospital $(\mathrm{BH})$ with AGE from January to September 2008*. To compare RV genotype between two consecutive winter seasons (2007 and 2008).

Methods: Prospective study of all children $\leq 16$ years, attending emergency room with AGE. A questionnaire and stool samples(SS) were collected. Stools were investigated for the presence of adenoviruses(AdV), rotaviruses(RV), noroviruses(NV) and enteroviruses(Echo) and were genotyped by reverse transcriptase-polymerase chain reaction.

Results: SS were collected from 207 children with median age of 17 months (1 month;16 years). In 83 $(40.1 \%)$ SS were isolated viruses (2 coinfection). RV was the most prevalent(18.8\%), followed by $\mathrm{NV}(11.6 \%)$. Adenoviruses and enteroviruses were identified in $7.2 \%$ and $3.4 \%$, respectively. NV was the most prevalent in July, August and September, RV was the most frequent detected in all other months. Five genotypes of $\mathrm{RV}$ were isolated, the most prevalent was G1P[8](43.6\%), followed by G9P[8](33.3\%). Two genotypes of NV were identify GII.4(95.8\%)$\mathrm{GI}(4.2 \%) ; 2 \mathrm{AdV}$ : AdV1(33.3\%)-AdV2(66.6\%) and 5 Echo genotypes:6(2),11,13,30,71.

Conclusions: RV wasthe mostfrequentisolatedvirus. G1P[8] was the most prevalent RV type detected. In 2007 G2P [4] was isolated in $77.8 \%$ of the samples, whereas in 2008 was present in only 1case. G9P[8] is still the second genotype identify $(1,9 \%$ vs $33,3 \%)$. These results show the variability of $\mathrm{RV}$ strains. This was the first study in Portugal with NV in AGE, we saw that $N V$ was the second most frequent agent and it was the most prevalent in the summer months. No enteric genotypes of AdV were identified.

*Started RVnational surveillance

302

\section{EFFECT OF HOME BASED CHILD CARE (HBCC) ON CHILD MORTALITY IN A TRIBAL POPULATION: RESULTS OF A FIELD TRIAL}

\section{A.R. Satav ${ }^{1,2}$}

${ }^{1}$ Tribal Health, MAHAN, Amaravati-Dharni, ${ }^{2}$ Medicine, Mahatma Gandhi Institute of Medical Sciences, Sevagram, Wardha, India

Background: Melghat is tribal area in India with very high child mortality \& malnutrition. The major causes of mortality \& morbidity are infectious diseases. We developed Home Based Child Care (HBCC) model for tribal population to reduce children mortality and infectious diseases.

\section{Objectives:}

1. To reduce Neonatal mortality rate (NMR), Infant Mortality rate (IMR) \& under 5 children mortality rate(U5MR) (especially due to infections) from 54, $90, \& 140$ to $32.4,58.05 \& 72.1$ per 1000 live births respectively in population of 14,120 of Melghat over 3 years.

2. To reduce incidence of infectious diseases by $35 \%$ over 3 years.

Methods: Study-design was Randomised Control Trial. We selected 16 intervention (population 14,888 ) and 18 control (population 16,310) villages. Trained village health workers in intervention area treated post-neonatal diseases such as diarrhoea, acute respiratory infections, malaria and neonatal diseases like neonatal sepsis, neonatal pneumonia, etc. Behaviour Change Communication programs were conducted.

Results: Baseline mortality indices in control versus intervention areas were: NMR- 57.19 vs 50.93, IMR- 72.97 vs 94.9, \& U5MR- 102.56 vs 143.52. After intervention NMR, IMR \& U5MR were significantly decreased in intervention area to $16.52,24.79 \& 37.19$ respectively $(p<0.05)$. The incidence, number of deaths \& case fatality rates due to infectious diseases in intervention area were reduced significantly $(p<0.05)$.

Conclusions: HBCC resulted in significant decrease in children mortality especially due to infections \& incidence of infectious diseases. Our model is replicable for reducing children mortality due to infectious disease. 